\title{
Assistência de Enfermagem frente a prevenção e ao tratamento dos pacientes acometidos por LPP em uma Unidade de Terapia Intensiva: Uma revisão sistemática com metassíntese
}

Nursing care front the prevention and treatment of patients affected by PPL in an Intensive Care Unit: A systematic review with metasynthesis

El cuidado de enfermería en la prevención y tratamiento de pacientes afectados por LPP en una Unidad de Cuidados Intensivos: Revisión sistemática con metasíntesis

José Willian Lima da Silva ORCID: https://orcid.org/0000-0003-2428-5082 Centro Universitário de Juazeiro do Norte, Brasil E-mail: josewillianlima12@gmail.com

Geovanna Renaissa Ferreira Caldas ORCID: https://orcid.org/0000-0001-9820-309X Centro Universitário de Juazeiro do Norte, Brasil E-mail: geovannacaldas@hotmail.com Angela Mendes Paes Campos ORCID: https://orcid.org/0000-0001-8960-8490

Centro Universitário Sudoeste Paulista, Brasil E-mail: camposmpangela@hotmail.com

Lohany Stéfhany Alves dos Santos ORCID: https://orcid.org/0000-0003-3440-740X Hospital Maternidade São Francisco de Assis, Brasil E-mail: lohanystefhany@gmail.com

Paloma Esterfanny Cardoso Pereira ORCID: https://orcid.org/0000-0003-4138-1201

Centro Universitário Santo Agostinho, Brasil E-mail: palomaesterfanny@hotmail.com

Ana Caren dos Santos Paz

ORCID: https://orcid.org/0000-0002-2766-1357

Universidade Estadual do Maranhão, Brasil

E-mail: ana.careen1@outlook.com

Maria Paloma Lima Sousa

ORCID: https://orcid.org/0000-0002-3029-0307 Secretaria Municipal de Saúde do Barro, Brasil

E-mail: paloma.lima2017@outlook.com

Renata Porangaba Cavalcante ORCID: https://orcid.org/0000-0002-9408-3251 UMJ- Centro Universitário Mario Pontes Juca, Brasil

E-mail: porangabarenata@gmail.com

Cicera Daniele Garcia Simões ORCID: https://orcid.org/0000-0001-6992-9860 Centro Universitário de Juazeiro do Norte, Brasil E-mail: danielegsousa@ hotmail.com

Laudilina Xavier Rocha Gonçalves ORCID: https://orcid.org/0000-0002-2352-7054 Centro Universitário de Juazeiro do Norte, Brasil E-mail: laudilinagonsalves@gmail.com

Carolina Francisca da Silva Ferreira ORCID: https://orcid.org/0000-0003-1955-0005 Centro Universitário de Juazeiro do Norte, Brasil E-mail: carollinadasilva@ hotmail.com

Dhiosen Berg da Silva Ferreira ORCID: https://orcid.org/0000-0001-9206-309X Faculdade SENSU, Brasil E-mail: dhiosenloirinha@hotmail.com

Janaina Cristina Sampaio Coelho Feitosa

ORCID: https://orcid.org/0000-0003-0832-9612 Hospital São Lucas, Brasil

E-mail: janacrisjcsc@hotmail.com 
Emanuel Cardoso Monte ORCID: https://orcid.org/0000-0002-4719-7168 Centro Universitário de Juazeiro do Norte, Brasil

E-mail: emanoelcm@gmail.com

Victor Augusto Moura ORCID: https://orcid.org/0000-0003-4239-5525 FENSG-UPE, Brasil E-mail: vito.moura1901@gmail.com

Cintia Nadhia Alencar Landim ORCID: https://orcid.org/0000-0002-8189-9323 Centro Universitário de Juazeiro do Norte, Brasil E-mail: nadhia_landim@hotmail.com

Maria Elisa Regina Benjamin de Moura ORCID: https://orcid.org/0000-0003-3145-4307 Centro Universitário de Juazeiro do Norte, Brasil E-mail: elisareg@gmail.com

Cicero Rafael Lopes da Silva ORCID: https://orcid.org/0000-0001-8819-5380 Centro Universitário de Juazeiro do Norte, Brasil E-mail: rafael.lopes@unijuazeiro.edu.br

\begin{abstract}
Resumo
O objetivo desse estudo foi analisar a assistência da equipe de enfermagem frente a prevenção e ao tratamento do paciente internado portador de Lesão por Pressão em Unidades de Terapia Intensiva. O presente artigo trata-se de uma revisão sistemática da literatura com metassíntese, que acompanha o protocolo PRISMA para a busca e utilização de estudos, dos quais, 22 artigos fizeram parte da construção do estudo. O levantamento das produções científicas ocorreu entre os meses de agosto de 2020 e setembro de 2020. Observou-se diante do estudo as dificuldades que o enfermeiro encontra na assistência dentro de uma UTI, desde a qualidade da assistência prestada, a dificuldade de implementação dos protocolos da unidade e até a falta de qualificação profissional. É necessária uma maior qualificação dos profissionais, e uma maior atenção aos pacientes em estado crítico da UTI, dessa forma podendo o enfermeiro agir de forma correta realizando uma assistência de forma qualificada.
\end{abstract}

Palavras-chave: Enfermagem; Lesão por pressão; Unidade de Terapia Intensiva.

\begin{abstract}
The aim of this study was to analyze the assistance of the nursing team regarding the prevention and treatment of inpatients with Pressure Injury in Intensive Care Units. This article is a systematic review of the literature with meta synthesis, which accompanies the PRISMA protocol for the search and use of studies, of which 22 articles were part of the construction of the study. The survey of scientific productions occurred between the months of August 2020 and September 2020. It was observed before the study the difficulties that nurses find in care within an ICU, from the quality of care provided, the difficulty of implementing the protocols of the unit and even the lack of professional qualification. It is necessary to have a higher qualification of the professionals, and a greater attention to patients in critical condition of the ICU, so that the nurse can act correctly, providing assistance in a qualified way.
\end{abstract}

Keywords: Nursing; Pressure injury; Intensive Care Unit.

\title{
Resumen
}

El objetivo de este estudio fue analizar la asistencia del equipo de enfermería en la prevención y tratamiento de pacientes hospitalizados con Lesión por Presión en Unidades de Cuidados Intensivos. Este artículo es una revisión sistemática de la literatura con meta-síntesis, que acompaña al protocolo PRISMA para la búsqueda y uso de estudios, de los cuales 22 artículos formaron parte de la construcción del estudio. La encuesta de producciones científicas ocurrió entre los meses de agosto de 2020 y septiembre de 2020. Se observó antes del estudio las dificultades que encuentran los enfermeros en la atención dentro de una UCI, desde la calidad de la atención brindada, la dificultad de implementar los protocolos de la unidad y incluso la falta de cualificación profesional. Es necesario contar con una mayor calificación de los profesionales, y una mayor atención a los pacientes en estado crítico de la UCI, para que el enfermero pueda actuar correctamente realizando una atención calificada.

Palabras clave: Enfermería; Lesión por presión; Unidad de Terapia Intensiva.

\section{Introdução}

O National Pressure Injury Advisory Panel (NPIAP)* em 2016, efetuou uma atualização para a terminologia da Úlcera por Pressão, que passou a ser referida de Lesão Por Pressão (LPP). Isso acontece pelo fato da lesão, não necessariamente precisa causar uma ferida profunda na pele, podendo ser de maneira superficial. Nessa nova nomenclatura a LPP vem a ser conceituada 
como uma lesão que será localizada na pele e podendo ser em estruturas com proeminências ósseas, que virá a ser resultante de uma pressão única ou sendo uma combinação com o cisalhamento ou a fricção (NPIAP 2016 \& Farias et al., 2019).

A lesão por sua possível derivação, desperta uma atenção maior para possíveis pontos que podem vir a se associar com essa derivação, levando a uma complicação do quadro clínico da lesão, entre alguns desses pontos, nós vamos identificar os dispositivos médicos em seu mau uso, os coxins quando não utilizado corretamente, até mesmo a permanência do decúbito prolongado em uma posição elevando a pressão na região (Adriani et al., 2019).

Pereira et al., 2019, vai demonstrar que quando se faz a abordagem na literatura em busca de estudos dentro da área, vai haver um grande número feito no exterior apresentando incidência de forma variada, dependendo da população utilizada na pesquisa. Já quando se adentramos nos estudos com foco hospitalar no Brasil, os 11Os números da incidência de LPP pode chegar a ter uma variação entre $20 \%$ e 59\%. Além desses dados, também se visualiza a elevação da prevalência, que vai decorrer do envelhecimento da população, que vai ter uma cascata de fatores a partir de doenças crônicas que podem levar a uma internação mais prolongada.

É válido ressaltar que as LPPs vêm se destacando como problema da saúde pública e por apresentar um fator de risco enorme para aquele paciente que está em situação de internação hospitalar. Tal fator de risco requer atenção do profissional de saúde, pois sem a intervenção devida poderá gerar um aumento do risco de infecção tanto pela ferida como pelo tempo de internação, intensificando o sofrimento do indivíduo. E além do paciente também temos as consequências que vão respingar na instituição quanto a carga de trabalho da equipe e custos hospitalares aumentados (Santos et al., 2020).

Durante a estadia do paciente na Unidade de Terapia Intensiva (UTI), existem situações que podem comprometer a perfusão tissular, fazendo a região virar propícia para uma lesão, como: redução da pressão sanguínea decorrente de alterações cardiovasculares, umidade da região, choque séptico, uso de fármacos e instabilidade hemodinâmica (Otto et al., 2019).

Portanto, a identificação dos fatores de risco se torna de fundamental importância para que ocorra uma implementação de ações curativas, porém não só curativas, mas profiláticas. Também se observa que a escala de Braden é o método mais utilizado para rastreio/avaliação do risco das LPPs, a escala se tornou a mais aplicada na rede hospitalar devido a sua confiabilidade e validades comprovadas em estudos realizados (Ribeiro et al., 2018).

O estudo será embasado na importância dada às LPPs até mesmo como um fator avaliativo no atendimento ofertado ao paciente. Compreendendo o atendimento ofertado e quais os riscos e problemas encontrados no tratamento que levam a incidência dessa doença, além de toda a importância do conteúdo abordado para o cotidiano dos profissionais que encaram essa lesão.

$\mathrm{Na}$ decorrência do que foi apresentado, tem-se como objetivo de estudo analisar a assistência da equipe de enfermagem frente a prevenção e ao tratamento do paciente internado portador de LPP em Unidade de Terapia Intensiva, avaliando todos os fatores que os pacientes vêm a ficar exposto durante todo esse processo.

\section{Metodologia}

O estudo em questão, trata-se de uma revisão sistemática da literatura com Metassíntese. A revisão sistemática vem a ser um tipo de desenho de investigação que acompanham protocolos específicos para a busca e utilização de estudos, buscando dar coerência ao seu corpus documental. Essa modalidade de pesquisa, é útil para agregar as informações de um conjunto de documentos socializados por vários autores sobre determinada temática, podendo apresentar resultados coincidentes ou opostos (Fiorentini, 2011).

A pergunta norteadora do estudo foi fundamentada de acordo com o acrônimo PICo (população, intervenção e contexto). Quais as ações de assistência de enfermagem para o paciente portador de LPP? Como se dá a assistência nas UTI? (P: População hospitalar; I: Atendimento em saúde; Co: Assistência realizada). 
Para alcançar a produção científica sobre a assistência de enfermagem aos pacientes portadores de LPP, foram utilizadas como fonte de busca a Biblioteca Virtual em Saúde (BVS) e as bases de dados: Scientific Electronic Library Online (SciELO), Medical Literature Analysis and Retrieval System Online (MEDLINE), Literatura Latino-americana e do Caribe em Ciências da Saúde (LILACS) e Base de Dados em Enfermagem (BDENF). O levantamento das produções científicas ocorreu entre os meses de julho de 2020 e outubro de 2020.

Durante a busca dos documentos, foram utilizados os Descritores em Ciências da Saúde (DeCS): "Enfermagem”, "Lesão Por Pressão" e "Unidade de Terapia Intensiva", associados ao operador booleano AND, da seguinte forma: "Enfermagem" AND "Lesão Por Pressão" AND "Unidade Terapia Intensiva".

Foram incluídos na pesquisa, os estudos que atendiam aos seguintes critérios empregados: $1^{\circ}$ ) artigos disponíveis integralmente, $2^{\circ}$ ) publicados entre os anos de 2018 a 2020, $3^{\circ}$ ) estarem escritos em português, inglês ou espanhol. Foram excluídos do estudo, os documentos que não possuíam como ideia central a assistência ao paciente acometido por LPP ou que não se enquadrava no contexto da UTI, estudos repetitivos em diferentes bases de dados, dissertações, documentários, monografias, projetos, resumos, relatos e teses.

Após a realização da leitura dos títulos e resumos de todos os materiais encontrados, foram selecionados os artigos que se enquadravam nos critérios de inclusão e exclusão definidos, para compor o estudo. No que tange aos aspectos éticos da pesquisa para as revisões, não se faz necessário a submissão ao Comitê de ética e pesquisa com seres humanos.

Uma vez que, os artigos utilizados já passaram pela a apreciação ética e encontram-se disponíveis online nas bases de dados. Assim, é possível afirmar que todos os preceitos da resolução do Conselho Nacional de Saúde nº 466/12 foram respeitados.

\section{Resultados e Discussões}

Ao realizar uma busca na BVS, foram filtradas apenas as bases de dados MEDLINE, LILACS E BDENF, foi obtido uma totalidade de 158 documentos. Ao realizar a mesma busca na SciELO, foram encontrados 01 estudos, somando um conjunto de 159 documentos distribuídos na MEDLINE (116); LILACS (27); SciELO (01) e BDENF (32). Apenas 115 estudos passaram pela triagem, 102 foram elegíveis e após aplicabilidade dos critérios de inclusão e exclusão, somente 22 artigos fizeram parte da construção do estudo.

Com o intuito de demonstrar o processo de busca e escolha dos estudos, foi utilizado um fluxograma baseado no protocolo PRISMA, ilustrado na Figura 1. 
Figura 1 - Fluxograma de eleição dos estudos.

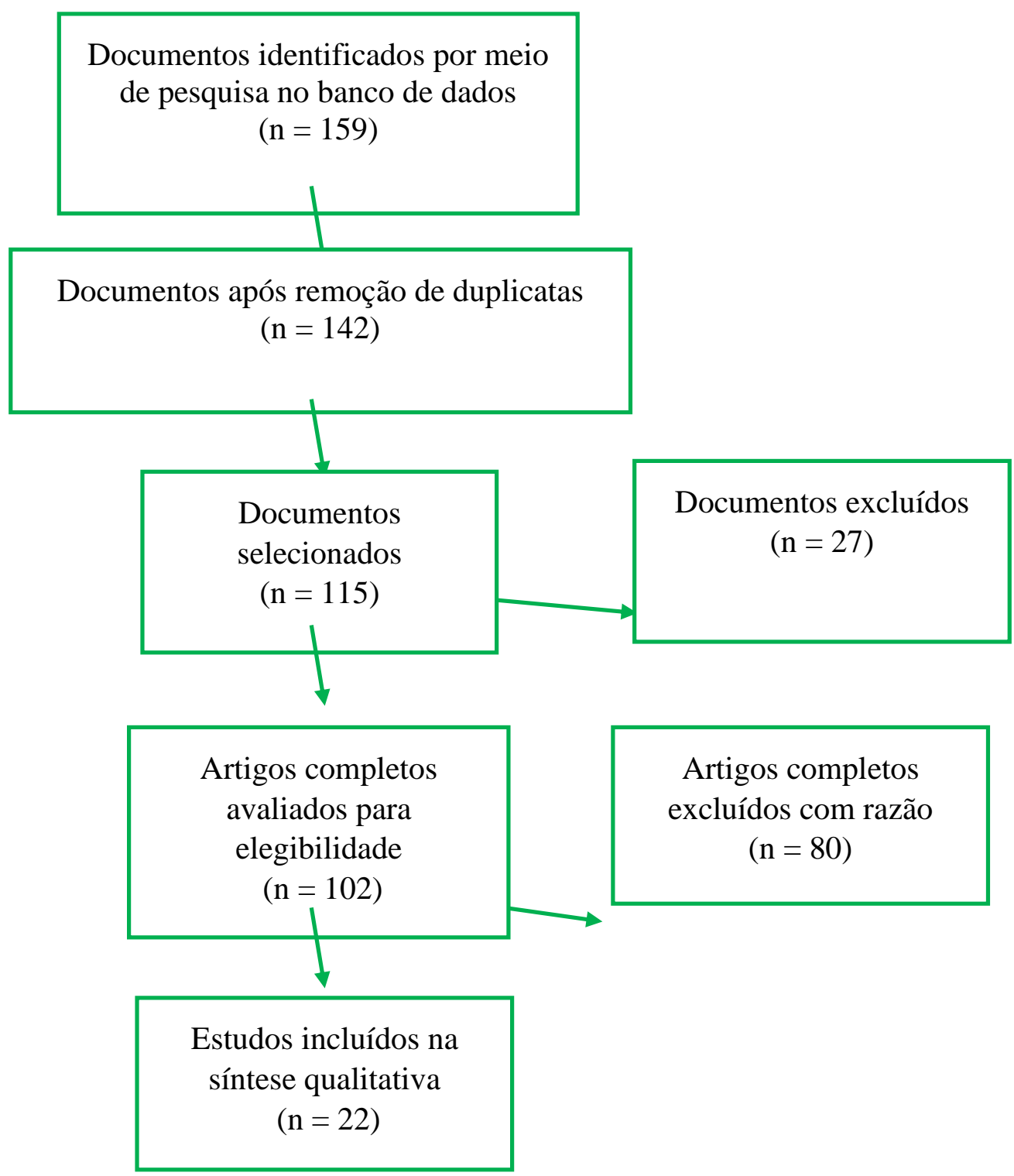

Fonte: Protocolo PRISMA.

Os documentos selecionados foram organizados de acordo com o achado mais recente, para o menos recente. Encontram-se ilustrados no Quadro 1, quanto ao autor e ano de publicação, objetivo e principais achados.

Quadro 1 - Distribuição dos artigos quanto ao autor e ano de publicação, objetivo e principais resultados.

\begin{tabular}{|l|l|l|}
\hline \multicolumn{1}{|c|}{ Autor e ano } & \multicolumn{1}{|c|}{ Objetivo } & Principais Resultados \\
\hline $\begin{array}{l}\text { Santos AJ, Oliveira LAG, } \\
\text { Paiva AA, Martins GM, } \\
\begin{array}{l}\text { Rodrigues KC, Oliveira } \\
\text { KCR, 2020 }\end{array}\end{array}$ & $\begin{array}{l}\text { Analisar o perfil de incidência de } \\
\text { lesões por pressão. }\end{array}$ & $\begin{array}{l}\text { Obteve-se que 57\% dos pacientes caracterizaram-se do sexo } \\
\text { masculino, 60,6\% na faixa etária maior ou igual a 60 anos, o tempo } \\
\text { média de permanência na unidade compreendeu 8 9 9,7 dias. O estudo } \\
\text { evidenciou baixa incidência de lesões por pressão nos pacientes da } \\
\text { unidade de terapia intensiva avaliada, levando-se em consideração o } \\
\text { referencial de estudos internacionais e nacionais. }\end{array}$ \\
\hline $\begin{array}{l}\text { Gonçalves ADC, Binda } \\
\text { ALM, Pinto EN, Oliveira } \\
\text { ESN, Binda I, 2020. }\end{array}$ & $\begin{array}{l}\text { Identificar os motivos que possam } \\
\text { estar relacionados à dificuldade da } \\
\text { realização da mudança de }\end{array}$ & $\begin{array}{l}\text { Enfermeiro é o profissional responsável não apenas } \\
\text { pelos cuidados assistenciais, mas pela avaliação dos fatores de } \\
\text { risco de LP na admissão, da integridade da pele e dos resultados } \\
\text { obtidos com as ações adotadas. foram encontradas falhas na execução }\end{array}$ \\
\hline
\end{tabular}




\begin{tabular}{|c|c|c|}
\hline & $\begin{array}{l}\text { decúbito de pacientes em terapia } \\
\text { intensiva. }\end{array}$ & $\begin{array}{l}\text { por falta de conhecimento, falha de integração entre os membros da } \\
\text { equipe, número deficiente } \quad \text { de pessoal ou } \\
\text { de protocolo para a padronização. }\end{array}$ \\
\hline $\begin{array}{l}\text { Ali YCMM, Souza TMP, } \\
\text { Garcia PC, Nogueira PC, } \\
2020\end{array}$ & $\begin{array}{l}\text { Correlacionar a incidência de } \\
\text { Lesão por pressão (LP) com o } \\
\text { tempo médio de assistência de } \\
\text { enfermagem em unidade de } \\
\text { terapia intensiva (UTI). }\end{array}$ & $\begin{array}{l}\text { Os resultados sugeriram sobrecarga da equipe. Confirmou a } \\
\text { importância da implementação e reavaliação da eficácia de protocolos } \\
\text { de cuidados preventivos para LP, além de alertar sobre a sobrecarga } \\
\text { de trabalho de enfermagem na assistência aos pacientes críticos }\end{array}$ \\
\hline Sales DO, Waters C, 2019 & $\begin{array}{l}\text { Identificar, a pontuação da Escala } \\
\text { de Braden nos pacientes adultos e } \\
\text { idosos internados em Unidade de } \\
\text { Terapia Intensiva e quais são as } \\
\text { medidas de prevenção de LPP } \\
\text { utilizadas na UTI. }\end{array}$ & $\begin{array}{l}\text { A maioria dos pacientes foi avaliada pela Escala de Braden com risco } \\
\text { moderado ou risco alto para desenvolver a LPP, apresentando escores } \\
\text { baixos na Escala de Braden. As medidas de prevenção para a LPP } \\
\text { estiveram relacionadas aos fatores intrínsecos e extrínsecos dos } \\
\text { pacientes, como mudança de decúbito, uso de colchões especiais e o } \\
\text { uso de coxins. }\end{array}$ \\
\hline $\begin{array}{l}\text { Roxa VDS, Ramos NM, } \\
\text { Domingos JEP, Pinheiro } \\
\text { JAA, Viana MCA, Bezerra } \\
\text { AM, } 2019\end{array}$ & $\begin{array}{l}\text { Avaliar o risco de } \\
\text { desenvolvimento de Lesão por } \\
\text { Pressão utilizando a escala de } \\
\text { Braden em pessoas sob cuidados } \\
\text { críticos em uma Unidade de } \\
\text { Terapia de Intensiva. }\end{array}$ & $\begin{array}{l}\text { Conforme a mobilidade, foi constatado que } 33,33 \% \text { dos pacientes } \\
\text { encontravam-se muito limitado e } 30 \% \text { completamente imóvel. Alguns } \\
\text { medicamentos utilizados durante a internação na UTI, principalmente } \\
\text { os de uso contínuo, podem colaborar para o aparecimento de lesão por } \\
\text { pressão, como os encontrados no presente estudo. Por exemplo, os } \\
\text { sedativos e analgésicos reduzem a percepção da dor, mas prejudicam } \\
\text { a mobilidade. }\end{array}$ \\
\hline $\begin{array}{l}\text { Pereira AA, França APFM, } \\
\text { Miranda AFAC, Rodrigues } \\
\text { EV, Guimarães JV, Rassy } \\
\text { EC, } 2019 .\end{array}$ & $\begin{array}{l}\text { Relatar o caso de pacientes que } \\
\text { utilizaram o colchão pneumático } \\
\text { e/ou piramidal como estratégia de } \\
\text { prevenção de lesões por pressão } \\
\text { em Unidade de Terapia Intensiva. }\end{array}$ & $\begin{array}{l}2(33,3 \%) \text { dos pacientes que foram colocados sob o uso de colchão } \\
\text { piramidal apresentaram lesão por pressão, enquanto dos } 4(66,67 \%) \\
\text { que utilizaram o colchão pneumático, apenas } 1(16,67 \%) \text { paciente } \\
\text { apresentou lesão. Estes dados demonstram dentro artigo, que uso do } \\
\text { colchão pneumático, quanto a prevenção de LPP é mais vantajoso que } \\
\text { o uso do colchão piramidal. }\end{array}$ \\
\hline $\begin{array}{l}\text { Otto C, Schumacher B, } \\
\text { Wiese LPL, Ferro C, } \\
\text { Rodrigues RA, } 2019 \text {. }\end{array}$ & $\begin{array}{l}\text { Identificar a relação entre os } \\
\text { fatores de risco para o } \\
\text { desenvolvimento de lesão por } \\
\text { pressão e determinar sua } \\
\text { incidência em pacientes críticos. }\end{array}$ & $\begin{array}{l}\text { A média de internação na UTI dos pacientes que desenvolveram LPP } \\
\text { foi de } 24,6 \text { dias e o tempo médio para o surgimento de LPP foi de } 2,7 \\
\text { dias. Também encontrando uma multicausalidade para a incidência de } \\
\text { LPP, independente do motivo de entrada do paciente. }\end{array}$ \\
\hline $\begin{array}{l}\text { Neiva MJLM, Sousa MM, } \\
\text { Silva MFN, Vieira JL, } \\
\text { Melo MF, Reis EM, } \\
\text { Ferreira MAL, Rocha LR, } \\
2019 .\end{array}$ & $\begin{array}{l}\text { Identificar ações de enfermagem } \\
\text { que visem prevenir as lesões por } \\
\text { pressão. }\end{array}$ & $\begin{array}{l}\text { Dentre as estratégias usadas estão desde o acolhimento do paciente na } \\
\text { unidade de saúde, com inspeção da pele, reavaliação diária a pacientes } \\
\text { totalmente acamados, até a realização de mudança de decúbito, } \\
\text { massagem de conforto, colchão especial e uso de coxins. Essa análise } \\
\text { mostra que é possível identificar e prevenir lesão por pressão, e que a } \\
\text { equipe de enfermagem é a principal responsável em prestar } \\
\text { assistência. }\end{array}$ \\
\hline $\begin{array}{l}\text { Farias ADA, Leal NTB, } \\
\text { Travassos NPR, Farias } \\
\text { AJA, Nobre AMD, } \\
\text { Almeida TCF, } 2019\end{array}$ & 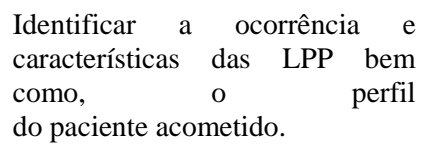 & $\begin{array}{l}\text { Identificou-se uma ocorrência de } 22,07 \% \text { com predomínio } \\
\text { de lesões em mulheres idosas, com distúrbios respiratórios e presença } \\
\text { de comorbidades. Sete pacientes já apresentavam a lesão na admissão } \\
\text { e } 10 \text { desenvolveram na UTI. }\end{array}$ \\
\hline $\begin{array}{l}\text { Adriani PA, Paggiaro AO, } \\
\text { Ferreira MC, Carvalho VF, } \\
2019\end{array}$ & $\begin{array}{l}\text { Identificar o nível de } \\
\text { conhecimento de enfermeiros } \\
\text { sobre a avaliação, prevenção e } \\
\text { classificação de lesões por pressão } \\
\text { em um hospital do Rio de Janeiro. }\end{array}$ & $\begin{array}{l}\text { Em relação aos resultados globais do teste, } 70 \%(68.63 \%) \text { dos } \\
\text { enfermeiros acertaram menos de } 70 \% \text { das questões, indicando um } \\
\text { déficit de conhecimento na área. Diante dos dados obtidos a partir } \\
\text { disso, identifica-se um déficit no conhecimento sobre a avaliação, } \\
\text { classificação e prevenção de LPP. }\end{array}$ \\
\hline $\begin{array}{l}\text { Teixeira LSA, Kawaguchi } \\
\text { MIAL, } 2019\end{array}$ & $\begin{array}{l}\text { Analisar os fatores de risco e o } \\
\text { tratamento da Lesão por Pressão } \\
\text { (LPP) em pacientes internados em } \\
\text { Unidades de Terapia Intensiva. }\end{array}$ & $\begin{array}{l}\text { Os resultados encontrados sugerem que uma avaliação inicial pela } \\
\text { equipe de enfermagem é a melhor forma para se prevenir a lesão por } \\
\text { pressão, e que os idosos estão mais propícios à lesão. Isso se torna } \\
\text { importante, pelo fato da LPP pode causar danos significativos ao } \\
\text { paciente acometido, podendo gerar um desconforto no processo de } \\
\text { recuperação. }\end{array}$ \\
\hline $\begin{array}{l}\text { Vargas RG, Santos LP, } \\
2019\end{array}$ & $\begin{array}{l}\text { Identificar os fatores de risco que } \\
\text { levam o desenvolvimento de } \\
\text { Lesão por pressão em pacientes } \\
\text { hospitalizados no setor de UTI. }\end{array}$ & $\begin{array}{l}\text { Os resultados apontaram que cuidados com a integridade da pele, } \\
\text { como a prevenção de lesões, constitui-se um importante elemento da } \\
\text { prática do enfermeiro, auxiliando na identificação de possíveis fatores } \\
\text { de risco que podem levar o desenvolvimento de lesões por pressão em } \\
\text { pacientes da UTI. Em síntese, medidas preventivas, tais como ações } \\
\text { de avaliação de risco e Escala de Braden auxiliam os profissionais para } \\
\text { prevenção de lesões por pressão. }\end{array}$ \\
\hline
\end{tabular}


Silva ACPMC, Ferreira AR, Coelho RR, Faria ID, 2019

Candaten AD, Vieira YB, Barcellos RA, 2019.

Cascão TRV, Rasche AS, Di Piero KC, 2019
Classificar os pacientes da amostra no momento da admissão no Centro de Terapia Intensiva quanto ao risco de desenvolverem LPP, além de levantar o quantitativo de lesões no momento da admissão.

Avaliar a incidência de lesões por pressão em um Hospital da Serra Outubro de 2017

Verificar a incidência de lesões por pressão na amostra estudada e identificar os fatores de risco para o desenvolvimento de lesões através da Escala de Braden em pacientes internados na Unidade de Terapia Intensiva de um Hospital Universitário no Rio de Janeiro.

Santos AFS, Oliveira BVB, Palmeira NMS, Carvalho SQS, Calheiros TRSP, França AMB, 2018 de Braden pelo enfermeiro na instituição hospitalar.
Avaliar a aplicabilidade da escala Gaúcha, no período de Janeiro a

Os fatores de risco mais apontados pela EB foram: atividade, nutrição e friç̧ão e cisalhamento. Os cuidados de enfermagem aos pacientes em risco de desenvolver lesões por pressão abrangem intervenções relacionadas ao acompanhamento integral do cliente. As escalas de avaliação de risco para LPP são instrumentos importantes, pois destacam pontos vulneráveis.

O surgimento das lesões por pressão, ainda é uma realidade durante a internação dos pacientes na UTI e o enfermeiro tem papel fundamental no planejamento das ações e cuidados de enfermagem, no tratamento e escolha de coberturas adequadas.

Paciente na UTI é superior a 10 dias. Ao investigarmos o tempo decorrido para o surgimento da primeira lesão constatamos que a maioria das LPP começaram a se desenvolver a partir do quinto dia de internação. Esse intervalo de tempo torna-se um fator de risco para o desenvolvimento desse tipo de lesão. Torna-se imprescindível a atuação do enfermeiro na linha de cuidados do paciente crítico na adoção precoce das medidas preventivas.

Analisa-se que apesar de ocorrer à avaliação de enfermagem através do protocolo, o tempo de internação do paciente influencia no desenvolvimento de lesão por pressão, ressaltando que os profissionais têm que verificar criteriosamente às sub-escalas para que o tratamento juntamente com a prevenção seja eficaz. Portanto, a escala de Braden, como outras escalas protocoladas nacionalmente, quando utilizadas de maneira conjunta, podem se tornar eficientes na assistência preventiva.

Identificar os principais fatores de risco para o desenvolvimento de lesão por pressão nesses ambientes no Brasil.

A UTI é pontuada como local propício para o desenvolvimento da LPP visto que os pacientes estão acamados e consequentemente com a mobilidade física prejudicada devido ao quadro clínico apresentado pelos mesmos e/ ou a terapêutica a que estão submetidos. O confinamento no leito e o número excessivo de dispositivos e tecnologias duras utilizadas, dificultam a mobilidade e a execução de manobras que proporcionem a prevenção da LPP, como a mudança de decúbito.

Sanches BO, Contrin LM, Beccaria LM, Frutuoso IS, Silveira AM, Werneck AL, 2018 enfermagem a o protocolo de lesão por pressão e segurança do paciente em unidades de terapia intensiva.

Tirgari B, Mirshekari L, Forouzi MA, 2018

Mendonça PK, Loureiro MDR, Junior MAF, Souza AS, 2018

Descrever a frequência e os fatores de risco para o desenvolvimento de Lesões por Pressão em clientes de Centros de Terapia Intensiva.

Examinar os conhecimentos e atitudes dos enfermeiros que atuam em unidades de terapia intensiva de hospitais afiliados à Universidade de Ciências Médicas Zahedanas em relação à prevenção de lesões por pressão.

Para determinar a incidência, taxa de incidência e fatores de risco de úlceras de pressão em pacientes de cuidados críticos.
Adesão da equipe de enfermagem ao protocolo, evidenciado por baixo índice de lesão quando comparado com a literatura, sendo que os pacientes do sexo masculino foram os que mais apresentaram. Comprovou-se associação estatística apenas com a idade, o que reflete a segurança dos pacientes quanto a prevenção de lesão por pressão em unidade de terapia intensiva.

A prevenção de lesões por pressão é uma das muitas prioridades do cuidado de enfermagem e é um indicador-chave da qualidade do cuidado de enfermagem. Para obter um atendimento de qualidade ideal nessa área, os gestores de enfermagem e outros administradores devem fazer esforços para melhorar os conhecimentos e atitudes de enfermagem com base nas mais recentes evidências científicas para a prevenção de lesões por pressão. Os resultados mostram deficiência quanto ao conhecimento de determinados protocolos para lhe dar com a LPP.

Dentre as variáveis estudadas, a idade foi igual ou maior a 60 anos, a presença de hipertermia e de pele edemaciada foram as que apresentaram significância estatística com a ocorrência de LP. Os fatores de risco identificados como significativos para a ocorrência de LP podem ser prevenidos, portanto, o envolvimento da equipe de enfermagem é fundamental na prevenção dessas lesões.

Complicações na unidade e no escore do prognóstico foram fatores de risco associados a úlceras de pressão, mas, surpreendentemente, o tempo de imobilização foi um fator protetor. identificação desses fatores pode ajudar os enfermeiros a estabelecer intervenções para prevenir úlceras de pressão neste cenário de saúde, uma vez que a 


\begin{tabular}{|l|l|l|}
\hline & & $\begin{array}{l}\text { prevenção de úlceras de pressão é um indicador da qualidade da } \\
\text { enfermagem. }\end{array}$ \\
\hline $\begin{array}{l}\text { Alencar GSA, Silva NM, } \\
\begin{array}{l}\text { Assis EV, Sousa MNA, } \\
\text { Pereira JLF, Oliveira WB, } \\
\text { Souza EF, 2018 }\end{array}\end{array}$ & $\begin{array}{l}\text { Identificar a incidência da LPP em } \\
\text { paciente internado na Unidade de } \\
\text { Terapia Intensiva e os fatores de } \\
\text { risco associados ao agravo. }\end{array}$ & $\begin{array}{l}\text { Percebeu-se uma maior probabilidade de desenvolvimento de LPP } \\
\text { após os } 60 \text { anos de idade, além dos pacientes que são submetidos a } \\
\text { procedimentos cirúrgicos. Infere-se que as LPP são agravos presentes } \\
\text { em unidades hospitalares que há pacientes em estado mais crítico, } \\
\text { devido à restrição permanente ou temporária ao leito. }\end{array}$ \\
\hline
\end{tabular}

Fonte: Autores.

Dessa forma, os 22 artigos utilizados para contextualizar os resultados, estão listados na Tabela 1, de acordo com a quantidade para cada ano de publicação.

Tabela 1 - Distribuição temporal dos artigos.

\begin{tabular}{cc}
\hline Ano de Publicação & Número de artigos \\
\hline 2018 & 7 \\
2019 & 12 \\
2020 & 3 \\
\hline TOTAL & $\mathbf{2 2}$ \\
\hline
\end{tabular}

Fonte: Autores.

Conforme Alencar et al., 2018, as Lesões Por Pressão (LPP) podem vir a ser caracterizadas como qualquer lesão causada por uma pressão não aliviada, cisalhamento ou fricção, que podem resultar em morte tecidual, sendo mais encontrada nas regiões que possuem proeminências ósseas.

Estas lesões podem estar relacionadas com alterações ou déficit nutricional, umidade, ventilação mecânica, alterações circulatórias, perfusão tissular alterada e principalmente, aumento da exposição à pressão, idade, sepses, período prolongado de hospitalização, como algumas doenças crônicas, entre elas diabetes, neuropatias e lesões medulares (Santos et al., 2018).

Para ser realizado uma prevenção adequada das LPP, se faz necessário compreender da melhor forma os fatores de risco que o paciente vai se encontrar sendo exposto no meio que está inserido, assim entendendo a melhor forma para prevenir os riscos de lesão para o mesmo (Caliri et al., 2016 \& Castanheira et al., 2018).

Quando falamos em assistência de enfermagem ao paciente acometido por essa patologia, dentro do contexto hospitalar, a UTI é o local mais propício para esse desenvolvimento, sendo consequentemente o local com maior cuidado e atenção na assistência, visto que no local se encontram pacientes acamados que tem uma mobilidade prejudicada, onde já se ver um fator de risco e predisposição ao surgimento de uma LPP (Bly, 2016).

Corroborando com a informação anterior, Sanches et al., 2018, demonstra que em UTI os pacientes apresentam alto risco para desenvolvimento de LPP por diminuição da percepção sensorial causada por sedativos, analgésicos e relaxantes musculares, determinando menor reação à pressão excessiva. Outros fatores contribuem como utilização de ventilador mecânico, cateter urinário, dispositivos de compressão sequencial, múltiplos cateteres intravenosos e infusão de drogas vasoativas.

Ainda na assistência, Sousa et al., 2019, vai dizer que o papel do enfermeiro vai ser determinante para o tratamento da lesão por pressão, pois é através da sistematização de enfermagem que o profissional vai prestar os cuidados integrais ao cliente de forma holística planejando as condutas clínicas e cuidados ao cliente, além da avaliação das intervenções e dos resultados obtidos, fazendo com que assim vem a acontecer um atendimento de qualidade na busca de um bom prognóstico.

Os profissionais envolvidos nessas situações, precisam estar atualizados e preparados para atuarem no processo da cicatrização, bem como atento para eliminação de fatores que possam vir a interferir no mesmo, o estudo de Roxa et al., 2019, 
demonstra que nos resultados finais, uma prevenção bem orientada, pode reduzir em até $60 \%$ o surgimento dessas lesões. Essa análise vai mostrar que se torna possível identificar e prevenir lesão por pressão, e que a equipe de enfermagem é a principal responsável em prestar essa assistência da maneira mais qualificada possível.

Neiva et al., 2019, no que rege as estratégias usadas, essas vão desde o acolhimento, como o tempo de permanência do paciente na unidade de saúde, é inspeção da pele principalmente durante o banho, sendo que esta pele deve ser limpa, hidratada e os lençóis secos e esticados para evitar lesões, e a importância da reavaliação diariamente principalmente paciente totalmente acamada, realizando e orientando sobre a importância da mudança de decúbito a cada 2 horas para aliviar a pressão em pontos das proeminências, usando também ferramentas na melhoria como colchão especial e uso de coxins.

Vindo em acréscimo ao que é dito anteriormente, Galvão e Ricarte, 2019, afirma que os registros das alterações de pele, a nutrição se encontra adequada para o estado do cliente, hidratação equilibrada e um meio importante para a prevenção, tratamento feito precocemente para pele antes de ocorrer a ruptura, além de monitorar e realizar as intervenções, mantendo tudo registrado, isso são algumas das ações que torna a assistência de enfermagem tão importante ao cuidado do paciente.

Além das técnicas utilizadas para a prevenção de LPP, Vargas e Santos, 2019, também se faz a aplicação de protocolos para se ter ciência do risco de cada paciente para desenvolver uma lesão, entre esses protocolos, temos a Escala de Braden. Tais instrumentos avaliativos, como a escala, tornam-se de suma relevância para determinação do risco de um paciente, uma vez que os valores baixos mostram maior propensão para desenvolver estas lesões. A avaliação diária deste risco pelo profissional enfermeiro, possibilita-o implementar ações individualizadas que minimizem a incidência deste agravo.

A enfermagem vai ter um papel fundamental e de grande responsabilidade no acompanhamento contínuo deste paciente, na prevenção e possível tratamento da LPP, visualizando que as lesões são evitáveis, Teixeira e Kawaguchi, 2019, demonstra em seus resultados que cabe a enfermagem e ao órgão hospitalar montar protocolo e colocar em prática escalas de avaliação de risco, como a já citada, escala de Braden. E até mesmo realizar metas, determinando um processo avaliativo contínuo, dessa forma preservando a integridade da pele, evitando maiores problemas para o paciente, família e própria equipe de enfermagem.

Dentro da assistência o profissional de enfermagem, junto de sua equipe, vai fazer necessário ter um cuidado maior aos fatores de risco de cada paciente, saber que o paciente idoso tem uma tendência maior a ser acometido por conta da frágil integridade da pele e a falta de locomoção, presença de edemas, obesidade, déficit nutricional, cisalhamento, circulação prejudicada e mobilidade diminuída, estão entre os fatores que o profissional deve estar sempre atento (Ribeiro et al., 2018).

Quando falamos da assistência feita na UTI, Farias et al., 2019, vai ressaltar que temos o fator de risco mais abundante, a mobilidade diminuída, por se tratar de pacientes críticos e na maioria dos casos entubados, não se há mobilidade, além da dificuldade na mudança de decúbito, uma escala de Braden bem elaborada e executada, vai nos mostrar o nível crítico do paciente nesse setor, fazendo com que ocorra uma assistência de maneira adequada, mantendo suas prioridades, nesse contexto se ver a importância e o quão auxiliador pode ser uma avaliação de qualidade.

Os estudos demonstram que, mesmo ocorrendo à avaliação de enfermagem por meio do protocolo, o tempo de internação influencia no desenvolvimento de LPP (Santos et al., 2018).

Pesquisadores apontam os desafios dos enfermeiros nas instituições hospitalares na prevenção de LPP, como: pressão que os profissionais sofrem durante a assistência, a carga de trabalho, a demanda elevada de pacientes com risco de desenvolvimento das lesões, além de não encontrar novas tecnologias para a sistematização dos dados dos pacientes e poucos estudos sobre a temática para promover uma educação continuada para o aperfeiçoamento dos enfermeiros e sua equipe (NEIVA et al., 2019).

Nessa perspectiva, nos estudos verifica-se que há uma preocupação dos profissionais de saúde com a prevenção de LPP, mesmo com realidades desfavoráveis dentro do setor de UTI hospitalar, além de se perceber que a escala de Braden, quando utilizada em conjunto com medidas adicionais, podem se tornar eficientes na assistência preventiva. 


\section{Conclusão}

Diante dos dados apresentados, é possível evidencia a necessidade de se trabalhar a sensibilização do comprometimento do profissional enfermeiro, mas não somente ele, os gestores e serviços também precisam estar atentos a isso, para que seja possível propiciar uma melhor condição de aquisição do conhecimento e desenvolvimento de competências, sempre estimulando o profissional a se atualizar e até buscar uma especialização em determinada área, seguindo o exemplo de enfermeiros dermatologistas e até estomoterapeutas, que se dedicam a ciência vindo com o domínio prestando o cuidado.

Contudo, a assistência anda em conjunto com o conhecimento se tornando importante trabalhar com especializações, uma vez que a enfermagem consegue ofertar essa condição aos profissionais, dessa forma estimulando os enfermeiros que estão na assistência direta no setor de UTI, semi-intensiva e clínicas, a ter esse olhar holístico e humanizado, específico da integridade tissular da pele.

Ressalta-se pôr fim a importância do profissional de enfermagem no acompanhamento do paciente acometido por LPP e no cliente em estado crítico, estando atento a todos os fatores de risco recorrentes e fazendo o uso dos protocolos da instituição, mantendo o olhar humanizado durante toda sua internação. Abrindo-se a porta para trabalhos futuros, onde esse ato seja mais observado e melhor avaliado de acordo com a evolução da área.

\section{Referências}

Adriani, P. A., Paggiaro, A. O., Ferreira, M. C., \& Carvalho, V. F. de. (2019). Aplicação do pressure ulcer knowledge test em enfermeiros de um hospital de atenção secundária - estudo transversal. Revista Enfermagem Atual In Derme, 87(25).

Alencar, G. S. A., Silva, N. M., Assis, E. V. et al. (2018). Lesão por pressão na unidade de terapia intensiva: incidência e fatores de risco. Revista Nursing, 21 (239): 2124-2128.

Bly, D. (2016). A model of pressure, oxygenation, and perfusion risk factors for pressure ulcers in the intensive care unit. American Journal of Critical Care, $25,156-164$.

Caliri, M. H. L., Santos, V. L. C. \& Gouveia, M. M. H. (2016). Publicação oficial da Associação Brasileira de Estomaterapia - SOBEST e da Associação Brasileira de Enfermagem em Dermatologia- SOBENDE 2016.

Castanheira, L. S., Alvarenga, A. W., Correa, A. R. et al. (2018). Escalas de predição de risco para lesão por pressão em pacientes criticamente enfermos: revisão integrativa.

Farias, A. D. A., Leal, N. T. B., Travassos, N. P. R. et al. (2019). Ocorrência de lesões por pressão em unidade de terapia intensiva de um hospital universitário. Revista Nursing, 2019; 22 (253): 2927-2931.

Fiorentini, D. (2011). Investigação em Educação Matemática desde a perspectiva acadêmica e profissional: desafios e possibilidades de aproximação. ANAIS do XIII Conferência Interamericana de Educação Matemática,CIAEM.

Galvão M. C. B. \& Ricarte I. L. M. (2019). Revisão sistemática da literatura: conceituação, produção e publicação.

National Pressure Injury Advisory Panel (2016). The National Pressure Injury Advisory Panel - NPIAP. About Us.

Neiva, M. J. L. M., Sousa, M. M., Silva, M. F. N. et al. (2019). Nursing care in prevention to pressure injuries in hospitalized patients. Braz. J. Hea. Rev., 2, 4336-4344.

Otto, C., Schumacher, B., Wiese, L. P. L. et al. (2019) Fatores de risco para o desenvolvimento de lesão por pressão em pacientes críticos. Enferm. Foco 2019; 10 (1): $07-11$.

Pereira, A. A., França, A. P. F. M., Miranda, A. F. A. C. et al. (2019). Prevenção de lesão por pressão e o uso do colchão pneumático versus colchão piramidal em unidade de terapia intensiva. Enferm Bras 2019;18(2):291-5.

Ribeiro, J. B., Santos, J. J., Fraga, I. M. N. et al. (2018). Principais fatores de risco para o desenvolvimento de lesão por pressão em unidades de terapia intensiva. Ciências Biológicas e de Saúde Unit, 5, 91-102.

Roxa, V. D. S., Ramos, N. M., Domingos, J. E. P. et al. (2019). Risk Assessment of Injury Pressure in Intensive Care Unit. Rev. Mult. Psic. 13, 946-960.

Sanches, B. O., Contrim, L. M., Beccaria, L. M. et al. (2018). Adherence of the nursing team to the protocols of pressure injury in intensive care unit. Arch. Health. Sci. 25(3) 27-31.

Santos, A. F. S., Oliveira, B. V. B., Palmeira, N. M. S. et al. (2018). A escala de braden como protocolo de prevenção de lesões por pressão: uma revisão integrativa. Ciências Biológicas e de Saúde Unit, 5, 193-204.

Santos, A. J., Oliveira, A. L. G., Paiva, A. A. et al. (2020). Incidência de lesões por pressão em unidade de terapia intensiva. Atenas Higeia 
Research, Society and Development, v. 10, n. 9, e10410917784, 2021

(CC BY 4.0) | ISSN 2525-3409 | DOI: http://dx.doi.org/10.33448/rsd-v10i9.17784

Sousa, R. G., Oliveira, T. R., Lima, L. R. et al. (2019). Fatores associados à úlcera por pressão (UPP) em pacientes críticos: revisão integrativa da literatura. Universitas: Ciências da Saúde. 2019;14(1):77-84.

Teixeira L. S. A. \& Kawaguchi, M. I. A. L. (2019). Prevenção e tratamento de lesões por pressão em pacientes internados na unidade de terapia intensiva. Revista JRG de Estudos Acadêmicos.

Vargas R. G. \& Santos, L. P. (2019). Prevenção de lesão por pressão em UTI - aplicabilidade da Escala de Braden. 